\title{
MATHEMATICAL MODEL OF THREE SPECIES FOOD CHAIN INTERACTION WITH MIXED FUNCTIONAL RESPONSE
}

\author{
MADA SANJAYA WS \\ Department of Mathematics, Faculty of Science and Technology, Universiti Malaysia \\ Terengganu, Kuala Terengganu,21030, Malaysia \\ Department of Physics, Faculty of Science and Technology, \\ Universitas Islam Negeri Sunan Gunung Djati, Bandung, Indonesia \\ madasws@gmail.com \\ ISMAIL BIN MOHD, MUSTAFA MAMAT and ZABIDIN SALLEH \\ Department of Mathematics, Faculty of Science and Technology, \\ Universiti Malaysia Terengganu, Kuala Terengganu,21030, Malaysia \\ ismailmd@umt.edu.my
}

\begin{abstract}
In this paper, we study mathematical model of ecology with a tritrophic food chain composed of a classical Lotka-Volterra functional response for prey and predator, and a Holling type-III functional response for predator and super predator. There are two equilibrium points of the system. In the parameter space, there are passages from instability to stability, which are called Hopf bifurcation points. For the first equilibrium point, it is possible to find bifurcation points analytically and to prove that the system has periodic solutions around these points. Furthermore the dynamical behaviors of this model are investigated. Models for biologically reasonable parameter values, exhibits stable, unstable periodic and limit cycles. The dynamical behavior is found to be very sensitive to parameter values as well as the parameters of the practical life. Computer simulations are carried out to explain the analytical findings.
\end{abstract}

Keywords: Food chain model; Lotka-Volterra model; Holling type-III functional response.

PACS numbers: $11.25 . \mathrm{Hf}, 123.1 \mathrm{~K}$

\section{Introduction}

Food chains in the environment are very important systems in many different fields such ecological science, applied mathematics, economic and engineering science. Food chains can be modeled by systems of differential equations which approximate species or functional feeding group behavior with different functional responses. 
Based on experiments (Refs. 1-3) suggested three different kinds of functional responses for different kinds of species to model the phenomena of predator, which made the standard Lotka-Volterra system more realistic. Biologically, it is quite natural the existence and asymptotical stability of equilibria and limit cycles for autonomous predator-prey systems with these functional responses.

Almost each of the food chain models considered in ecological literature are constructed by invoking same type of functional responses for prey-predator and predator-super predator interactions. But a different selection of functional response would be perhaps more realistic in this context. From this point of view, we have considered a classical (nonlogistic) Lotka-Volterra functional response for the species prey and predator and a Holling type-III functional response for the species predator and super predator.

\section{Mathematical Models}

The classical food chain models with only two trophic levels are shown to be insufficient to produce realistic dynamics. ${ }^{4,5,6,7}$ Therefore, in this paper, we consider the following three species food chain interaction. With non dimensionalization, the system of three species food chain can be written as

$$
\left.\begin{array}{l}
\frac{d x}{d t}=\left(a_{1}-b_{1} y\right) x \\
\frac{d y}{d t}=\left(-a_{2}+b_{2} x-\frac{c_{1} z y}{D^{2}+y^{2}}\right) y \\
\frac{d z}{d t}=\left(-a_{3}+\frac{c_{2} y^{2}}{D^{2}+y^{2}}\right) z
\end{array}\right\}
$$

where $x, y$, and $z$ denote the non dimensional population density of the prey, predator, and super predator respectively. The predator $y$ preys on $x$ and the predator $z$ preys on $y$. Furthermore, $a_{1}, a_{2}, a_{3}, b_{1}, b_{2}, c_{1}, c_{2}$ and $D$ are the intrinsic growth rate of the prey, the death rate of the predator, the death rate of the super predator, predation rate of the predator, the conversion rate, the maximal growth rate of the predator, conversion factor, and the half saturation constant respectively.

\subsection{Equilibrium point analysis}

The equilibrium points of (1) denoted by $E(\bar{x}, \bar{y}, \bar{z})$, are the zeros of its nonlinear algebraic system ${ }^{8,9}$ which can be written as

$$
\left.\begin{array}{rl}
\left(a_{1}-b_{1} y\right) x & =0 \\
\left(-a_{2}+b_{2} x-\frac{c_{1} z y}{D^{2}+y^{2}}\right) y & =0 \\
\left(-a_{3}+\frac{c_{2} y^{2}}{D^{2}+y^{2}}\right) z & =0
\end{array}\right\}
$$


By considering the positivity of the parameters and the unknowns, we have two positive equilibrium points given by $E_{0}(0,0,0)$, and $E_{1}\left(x_{1}, y_{1}, 0\right)$ with

$$
x_{1}=\frac{a_{2}}{b_{2}}, \quad \text { and } \quad y_{1}=\frac{a_{1}}{b_{1}}
$$

\subsection{Stability of equilibrium point}

The dynamical behavior of equilibrium points can be studied by computing the eigenvalues of the Jacobian matrix, $J$ of system (1) where

$$
\left[\begin{array}{ccc}
a_{1}-b_{1} \bar{y} & -b_{1} \bar{x} & 0 \\
b_{2} \bar{y} & -a_{2}+b_{2} \bar{x}-\frac{2 c_{1} y D^{2} \bar{z}}{\left(D^{2}+\bar{y}^{2}\right)^{2}} & -\frac{c_{1} \bar{y}^{2}}{\left(D^{2}+\bar{y}^{2}\right)} \\
0 & \frac{2 c_{2} y D^{2} b a r z}{\left(D^{2}+\bar{y}^{2}\right)^{2}} & a_{3}+\frac{c_{2} \bar{y}^{2}}{D^{2}+\bar{y}^{2}}
\end{array}\right]
$$

At most, there exists two positive equilibrium points for system (1). The existence and local stability conditions of these equilibrium points are as follows.

(1) The Jacobian matrix (3) at the equilibrium point $E_{0}(0,0,0)$, is

$$
J(0,0,0)=\left[\begin{array}{ccc}
a_{1} & 0 & 0 \\
0 & -a_{2} & 0 \\
0 & 0 & -a_{3}
\end{array}\right]
$$

The eigenvalues of the Jacobian matrix (4) are $\lambda_{1}=a_{1}, \lambda_{2}=-a_{2}$ and $\lambda_{3}=-a_{3}$. Hence, the equilibrium point $E_{0}$ is a saddle point.

(2) The Jacobian matrix (3) at the equilibrium point $E_{1}\left(x_{1}, y_{1}, 0\right)$, is

$$
J\left(x_{t}, y_{t}, 0\right)=\left[\begin{array}{ccc}
0 & -\frac{b_{1} a_{2}}{b_{1}} & 0 \\
\frac{b_{2} a_{1}}{b_{1}} & 0 & -\frac{c_{1} a_{1}^{2}}{b_{1}^{2}\left(D^{2}+\frac{a_{1}^{2}}{b_{1}^{2}}\right)} \\
0 & 0 & -a_{3}+\frac{c_{2} a_{1}^{2}}{b_{1}^{2}\left(D^{2}+\frac{a_{1}^{2}}{b_{1}^{2}}\right)}
\end{array}\right]
$$

The eigenvalues of the Jacobian matrix (5) are

$$
\left.\begin{array}{r}
\lambda_{1,2}= \pm i \sqrt{a_{1} a_{2}} \\
\lambda_{3}=\frac{c_{2} a_{1}^{2}-a_{3} b_{1}^{2} D^{2}-a_{3} a_{1}^{2}}{b_{1}^{2} D^{2}+a_{1}^{2}}
\end{array}\right\}
$$

$E_{1}\left(x_{1}, y_{1}, 0\right)$ is locally and asymptotically stable if

$$
a_{3} a_{1}^{2}+a_{3} b_{1}^{2} D^{2}>c_{2} a_{1}^{2}
$$




\subsubsection{Hopf Bifurcation Point}

When we are interested to study periodic or quasi periodic behavior of a dynamical system, we need to consider the Hopf bifurcation point. The dynamical system generally can be written as

$$
\begin{gathered}
\dot{v}=F(v, u) \\
\dot{v}=F(v, u) \text { diseqn }
\end{gathered}
$$

where

$$
v=(x, y, z), \quad \mu=\left(a_{1}, a_{2}, a_{3}, b_{1}, b_{2}, c_{1}, c_{2}\right)
$$

The system (1) can be written in the form (8)-(10), if an ordered pair $\left(v_{0}, \mu_{0}\right)$ satisfied the conditions

(1) $F\left(v_{0}, \mu_{0}=0\right.$

(2) $J(v, \mu)$ has two complex conjugate eigenvalues

$$
\lambda_{1,2}=a(v, \mu) \pm i b(v, \mu)
$$

around $\left(v_{0}, \mu_{0}\right)$

(3) $a\left(v_{0}, \mu_{0}\right)=0, \nabla a\left(v_{0}, \mu_{0}\right) \neq 0, b\left(v_{0}, \mu_{0}\right) \neq 0$, and

(4) the third eigenvalues $\lambda_{3}\left(v_{0}, \mu_{0}\right) \neq 0$, then $\left(v_{0}, \mu_{0}\right)$ is called a Hopf bifurcation point. ${ }^{8,9}$

For the system (1), the equilibrium points $E_{0}(0,0,0)$ and $E_{1}\left(x_{1}, y_{1}, 0\right)$, satisfy the condition $F\left(v_{0}, \mu_{0}\right)=0$, and for the equilibrium point $E_{1}\left(x_{1}, y_{1}, 0\right)$, we have two complex conjugate eigenvalues (6) with the real part of the eigenvalues are zero. The last condition $\lambda_{3}\left(v_{0}, \mu_{0}\right) \neq 0$ is satisfied if

$$
\begin{gathered}
a_{3} a_{1}^{2}+a_{3} b_{1} D^{2} \neq c_{2} a_{1}^{2} . \\
a_{30}=\frac{a_{1}^{2} c_{2}}{a_{1}^{2}+b_{1}^{2} D^{2}} .
\end{gathered}
$$

\section{Numerical Simulation}

Analytical studies always remain incomplete without numerical verification of the results. In this section, we present numerical simulation to illustrate the results obtained in previous sections. The numerical experiments are designed to show the dynamical behavior of the system in three main different sets of parameters: I. The case $a_{3}<a_{30}$, II. The case $a_{3}=a_{30}$, III. The case $a_{3}>a_{30}$. The coordinates of equilibrium points and the corresponding eigenvalues are can be found in Table 1. For showing the dynamics of the system (2) change, the parameter set $\left\{a_{1}, a_{2}, b_{1}, b_{2}, c_{1}, c_{2}, D\right\}=0.5,0.5,0.5,0.5,0.6,0.75,10$ as a fixed parameters and $a_{3}$ as a varied parameters. The calculation for the parameter set given Hopf bifurcation point (11) $a_{30}=0.007426$ as a reference parameter. 


\subsection{The case $a_{3}<a_{30}$}

For the case $a_{3}<a_{30}$ (see Table 1 ) two eigenvalues for E1 is pure imaginary initially spiral stability corresponding with center manifold in $x y$-plane and one positive real eigenvalues corresponding with unstable one dimensional invariant curve in $z$-axes. Hence the equilibrium point $E_{1}$ is a locally unstable spiral source and E0 is saddle points with real eigenvalues have opposite sign. In this case the super predator $\mathrm{z}$ can survive, growing periodic unstable. On the other hand, prey $x$ and predator $y$ persist and has populations that vary asymptotically stable. The plot of the solution in Fig. 1, exhibits this behavior.

\subsection{The case $a_{3}=a_{30}$}

For the case $a_{3}=a_{30}$ the equilibrium E1 has three eigenvalues with zero real part corresponding with stable center point in $x y$-plane (see Table 1 ). In this case super predator growth stable periodic. On the other hand, prey $x$ and predator $y$ persist

Table 1. Numerical experiment of stability equilibrium point

\begin{tabular}{|c|c|c|c|c|c|}
\hline The Case & Parameters & $\begin{array}{c}\text { Equilibrium } \\
\text { Point }\end{array}$ & & Eigenvalues & \\
\hline & & $E_{0}$ & $E_{1}$ & $E_{0}$ & $E_{1}$ \\
\hline$a_{1}<a_{30}$ & $a_{3}=0.005$ & $0,0,0$ & $1,1,0$ & $\pm 0.5,-0.005$ & $\pm 0.5 i, 0.002426$ \\
$a_{1}=a_{30}$ & $a_{3}=0.007426$ & $0,0,0$ & $1,1,0$ & $\pm 0.5,-0.007426$ & $\pm 0.5 i, 0$ \\
$a_{1}>a_{30}$ & $a_{3}=0.05$ & $0,0,0$ & $1,1,0$ & $\pm 0.5,-0.05$ & $\pm 0.5 i,-0.042574$ \\
\hline
\end{tabular}
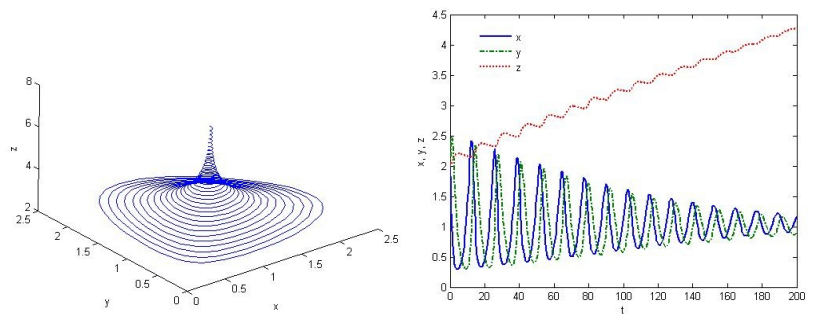

Fig. 1. The solution for $a_{3}<a_{30}$ : phase space and population time series for $a_{3}=0.005$.
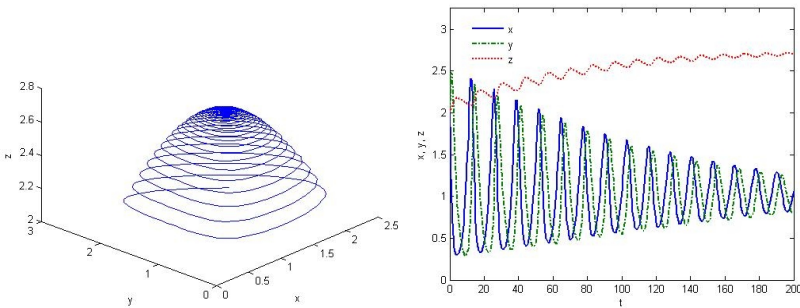

Fig. 2. The solution for $a_{3}=a_{30}$ : phase space and population time series for $a_{3}=0.007426$. 

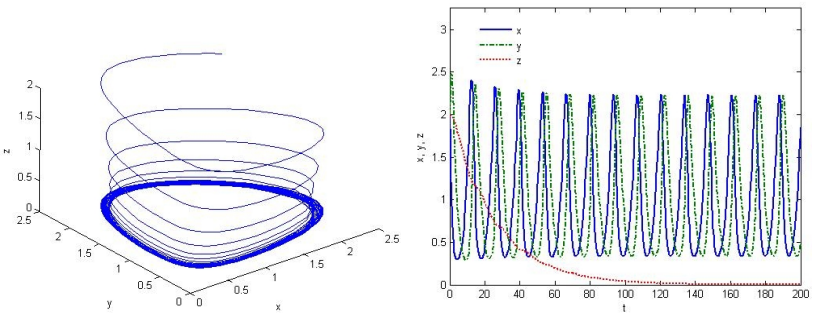

Fig. 3. The solution for $a_{3}>a_{30}$ : phase space and population time series for $a_{3}=0.05$.

and has populations that vary asymptotically stable. The plot of the solution in Fig. 2. exhibits this behavior.

\subsection{The case $a_{3}>a_{30}$}

For the case $a_{3}>a_{30}$ (see Table 1 ) two eigenvalues for E1 is pure imaginary initially spiral stability corresponding with center manifold in $x y$-plane and one negative real eigenvalues corresponding with stable one dimensional invariant curve in $z$ axes. Hence the equilibrium point E1 is locally stable spiral sink and $E_{0}$ is a saddle point with real eigenvalues have opposite sign. In this case super predators die. On the other hand, prey $x$ and predator $y$ persist and has populations that vary asymptotically stable. The plot of the solution in Fig. 3, exhibits this behavior.

The persistence of super predator species $z$ in (1) depends on the parameters $a_{1}, a_{3}, b_{1}, c_{2}$ and D. In particular, if $a_{3}>a_{30}$, then species $\mathrm{z}$ decrease over time to dies out, while if $a_{3} \leq a_{30}$, then species $z$ survives. On the other hand species prey $x$ and middle predator $y$ can persist for all condition with stable asymptotically.

\section{Conclusion}

In this paper, mathematical ecological models a tritrophic food chain with mixed functional response are studied. In this paper, three species food chain model are analyzed and possible dynamical behavior of this system is investigated at equilibrium points. It has been shown that, the solutions posses Hopf bifurcations, as one parameter is varied, the dynamics of the system change from a stable to a center to unstable. Both analytically and numerically simulation shown that in certain regions of the parameter space, the model is sensitively depending on the parameter values and initial condition.

\section{References}

1. C. S. Holling, The functional response of predator to prey density and its role in mimicry and population regulation, Mem. Ent. Sec. Can. 45, 1-60 (1965).

2. Z. Shuwen and C. Lansun, A Holling II functional response food chain model with impulsive perturbations, Chaos, Solitons $\&$ Fractals 24, 1269-1278 (2005). 
3. Z. Shuwen and T. Dejun, Permanence in a food chain system with impulsive perturbations, Chaos, Solitons \& Fractals 40, 392-400 (2009).

4. S. B. Hsu, T. W. Hwang and Y. Kuang, A ratio-dependent food chain model and its applications to biological control, Mathematical Biosciences 181, 55-83 (2003).

5. H. I. Freedman and P. Waltman, Mathematical analysis of some three species foodchain models, Math. Biosci. 33, 257 (1977).

6. A. Hastings and T. Powell, Chaos in a three-species food chain, Ecology 72, 896 (1991).

7. A. Hastings and A. Klebanoff, Chaos in three species food chains, J.Math. Biol. 32, 427 (1993).

8. H. C. Hilborn, Chaos and Nonlinear Dynamics (Oxford University Press, New York, 1994).

9. R. M. May, Stability and Complexity in Model Ecosystems (Princeton University Press, Princeton NJ, 2001). 